\title{
Radiographic Measurement of Cochlear Duct Length in an Indian Cadaveric Population - Importance of Custom Fit Cochlear Implant Electrodes
}

\author{
Anup Singh ${ }^{10}$ Rajeev Kumar ${ }^{1}$ Smita Manchanda ${ }^{2}$ Ashu Seith Bhalla ${ }^{2}$ Prem Sagar $^{1}$ \\ David Victor Kumar Irugu ${ }^{1}$
}

${ }^{1}$ Department of Otolaryngology and Head and Neck Surgery, All India Institute of Medical Sciences, New Delhi, India

${ }^{2}$ Department of Radiodiagnosis, All India Institute of Medical

Sciences, New Delhi, India

Address for correspondence Rajeev Kumar, MS-ENT, Department of Otolaryngology and Head and Neck Surgery, All India Institute of Medical Sciences, Room No.-4057, 4th Floor, Teaching Block, New Delhi-110029, India (e-mail: rajeev9843@yahoo.co.in).

Int Arch Otorhinolaryngol 2020;24(4):e492-e495.

\begin{abstract}
Keywords

- cochlear implantation

- cochlear duct

- tomography

- adult

- cadaver

Introduction Successful cochlear implantation requires an appropriate insertion depth of the electrode, which depends on cochlear duct length $\mathrm{CDL}$ ). The CDL can vary due to ethnic factors.

Objective The objective of the current study was to determine the CDL in an Indian adult cadaveric population.

Methods The present was a cadaveric study using the temporal bones obtained after permission of the Institutional Review Board. The temporal bones were subjected to high-resolution computed tomography (HRCT), and the double oblique reformatted $\mathrm{CT}$ images were reconstructed through the basal turn of the cochlea. The reformatted images were then viewed in the minimum-intensity projection (minIP) mode, and the ' $A$ ' value (the diameter of the basal turn of the cochlea) was calculated. The CDL was then measured using the formula $\mathrm{CDL}=4.16 \mathrm{~A}-4$ (Alexiades et al). The data analysis was performed using the Microsoft Excel software, version 2016.

Results A total of 51 temporal bones were included for imaging analysis. The CDL varied from $27.6 \mathrm{~mm}$ to $33.4 \mathrm{~mm}$, with a mean length of $30.7 \mathrm{~mm}$. There was no statistically significant difference between the two sides.

Conclusion The CDL can be calculated with preoperative high-resolution CT, and can provide a roadmap for effective cochlear implant electrode insertion. The populationbased anatomical variability needs to be taken into account to offer the most efficient and least traumatic insertion of the electrode.
\end{abstract}

\section{Introduction}

Cochlear ductal length (CDL) refers to the length of cochlea measured as the spiral distance from round window to the helicotrema. Measurements of the CDL have been made as early as $1865 .{ }^{1}$ The initial studies to report on this parameter were histological studies. ${ }^{1-3}$ With time and the evolution of technology, various techniques, including thin reformatted

received

October 6, 2018

accepted

November 20, 2019
DOI https://doi.org/

$10.1055 / \mathrm{s}-0040-1701272$. ISSN 1809-9777. high-resolution computed tomography (HRCT) scan cuts with 3-D reconstruction of the temporal bone are now being used to estimate the CDL. The values of these investigations have been compared with the histological findings of previous studies, and a good correlation between them has been obtained.

The CDL estimation holds importance during the insertion of electrodes in cochlear implant surgeries. Especially

Copyright $\odot 2020$ by Thieme Revinter Publicações Ltda, Rio de Janeiro, Brazil

License terms

(1) $\ominus \circledast$ 
with the advent of residual hearing preservation techniques, the precise insertion of electrodes to a certain depth to avoid damaging residual hearing cannot be overemphasized. The tactile force feedback during implant insertion cannot be solely relied upon to avoid insertional trauma, and a preoperative determination of the length of the cochlear duct will help in the insertion of an accurate and desirable electrode length. The CDL has been shown to harbor gender and ethnic variability. In the current study, we estimated the CDL in Indian cadaveric temporal bones using HRCT.

\section{Materials and Methods}

The present was a radiological study of human cadaveric temporal bones conducted at a tertiary care center in Northern India. Cadaveric temporal bones were obtained from unidentified/unclaimed cadavers from the Department of Forensic Medicine and Toxicology. The approval for the study was obtained from the institutional ethics committee.

The fresh cadaveric temporal bones were stored in $10 \%$ formalin and were subjected to imaging in the form of HRCT.

\section{Imaging Protocol}

\section{Image Acquisition and Reconstruction}

The CT scans were acquired in the HRCT temporal-bone protocol on a 40 slice CT Scanner (Sensation 40, Siemens, Erlangen, Germany). From this dataset, thin slice images $(0.6 \mathrm{~mm})$ were reconstructed in the axial plane in high resolution bone algorithm.

\section{Image Transfer and Analysis}

These images were then transferred to the advanced viewing workstation (Syngovia, Siemens, Erlangen, Germany) for advanced analysis and to the Picture Archiving and Communication Systems (PACS) (Syngoplaza, Siemens, Erlangen, Germany) for permanent storage.

\section{Interpretation}

Each dataset was then opened in the multiplanar reconstruction mode.

Double oblique reformatted CT images were reconstructed through the basal turn of cochlea.

The reformatted image was then viewed in the minimumintensity projection mode (minIP), with thickness varying from $1.3 \mathrm{~mm}$ to $1.5 \mathrm{~mm}$ to visualize the cochlear turns.

The variable $A$ was measured as the linear measurement from the center point of round window to the farthest point on the opposite wall of the cochlea passing through modiolus (-Fig. 1).

All of these values were saved as screenshots and then archived again in the PACS system.

The radiologically obtained ' $A$ ' value was used to calculate the cochlear length using the equation proposed by Alexiades et $\mathrm{al}^{4}(\mathrm{CDL}=4.16 \mathrm{~A}-4)$. The data was entered and analyzed using the Microsoft Excel (Microsoft Corp. Redmond, WA, US) software, version 2016.

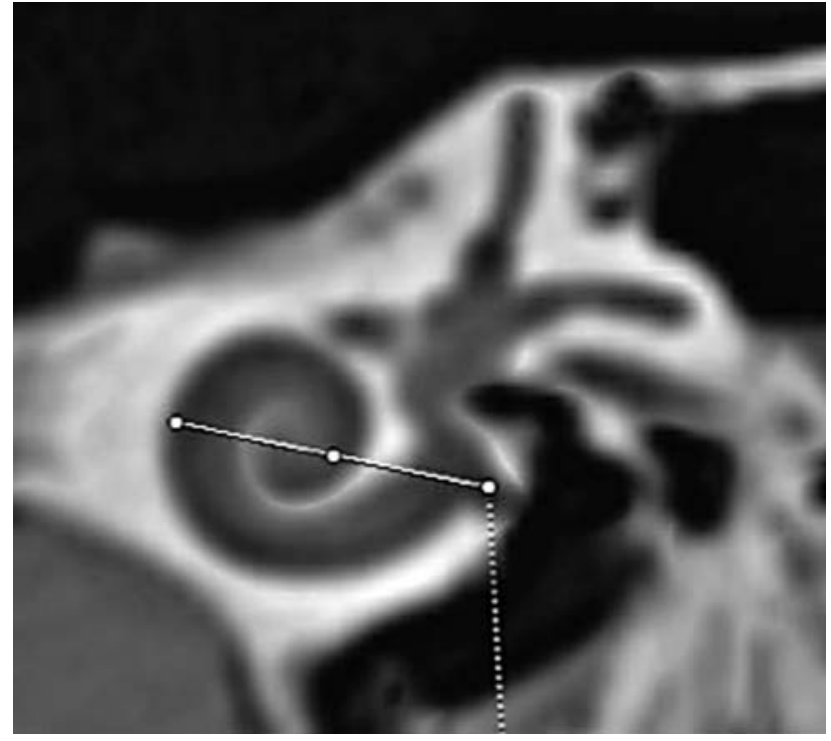

Fig. 1 Measurement of the variable ' $A$ ' in double oblique reformatted computed tomography (CT) images. The variable ' $A$ ' was measured from the center of the round window to the farthest point on the opposite wall of the cochlea passing through the modiolus in the minimum-intensity projection (minIP) mode of the reformatted image.

\section{Results}

A total of 51 adult cadaveric temporal bones with normal cochleovestibular morphology (on HRCT temporal bone scans) were included in the study. Information on gender could not be obtained. In total, there were 26 right-sided and 25 left-sided bones. The CDL varied from $27.6 \mathrm{~mm}$ to $33.4 \mathrm{~mm}$, with a mean of $30.7 \mathrm{~mm}$ and standard deviation (SD) of 1.66 . The mean cochlear length on the right side was of $30.5 \mathrm{~mm}$ (SD: 1.59), and, on the left side, it was of $30.8 \mathrm{~mm}$ (SD: 1.74). There was no statistically significant difference between the CDL on the two sides (unpaired $t$-test; $p=0.52$ ). - Fig. 2 shows the graphical distribution of the CDLs on right and left sides.

\section{Discussion}

With the introduction of hybrid technology or electro-acoustic stimulation (EAS), the precise insertion of the electrode inside the cochlea to achieve acoustic perception at high frequencies, while avoiding deeper insertions to avoid intracochlear trauma, has become of paramount importance. Speech perception depends on pitch-matching between the frequency stimulation being delivered by the cochlear implant electrode and the tonotopically mapped area of the cochlea receiving the stimulus.

The human cochlea is fully formed at birth, and the length of the human cochlear duct has been traditionally considered to be roughly $34 \mathrm{~mm} .{ }^{5}$ However, the distribution of the CDL in the population shows a significant range, and variability has been observed due to gender and the ethnic factors. ${ }^{6-10}$

The first documented record of CDL estimation was made by Hensen in 1865 by direct histologic measurements at the location of the organ of Corti using micrometers (cited from 


\section{Graphical representation of cochlear duct length}

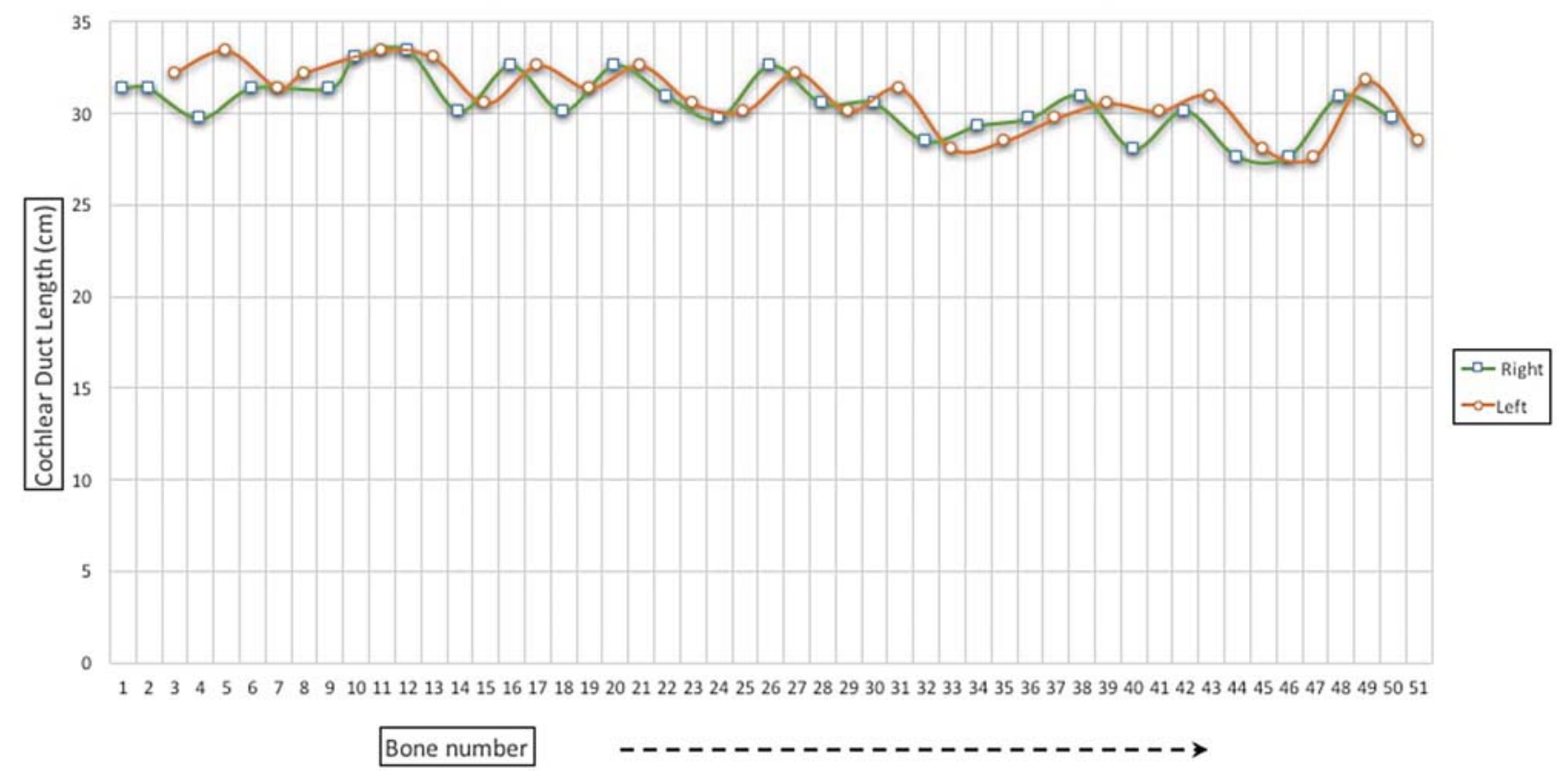

Fig. 2 Graphical distribution of the high-resolution CT scan-derived cochlear duct length on the right and left sides of the cadaveric temporal bones. ( $x$-axis number of temporal bones; $y$-axis - cochlear duct length in cm; green line - right side temporal bones; red line -left side temporal bones).

Hardy ${ }^{1}$ ). The length was determined to be $33.5 \mathrm{~mm}$ in this case. However, the first landmark study using indirect methods to plot a two-dimensional (2D) graphical representation of the histologic sections was published by Hardy ${ }^{1}$ in 1938 in 68 samples. The mean length was of $31.52 \mathrm{~mm}$, and the variation was of around $10 \mathrm{~mm}$. Another indirect method used a plastic cast of the cochlea to measure the length of the lateral wall of the cochlea. ${ }^{10}$ The most accurate method of CDL measurement has been the three-dimensional (3D) reconstruction of points on histologic sections or CT scan data. ${ }^{11}$ However, the apparent practical difficulty was due to the time taken to reconstruct the 3D image using histologic sections. ${ }^{12,13}$ The most recent method has been the use of the so-called ' $A$ ' value (the farthest distance from the round window center to the opposite lateral wall passing through the modiolus) by Escude et $\mathrm{al}^{14}$ to calculate the length of the basal turn. The length of the cochlea, which resembles an Archimedean spiral, made it possible to derive a spiral coefficient. Alexiades et $\mathrm{al}^{4}$ integrated the data published by Hardy ${ }^{1}$ into the calculation of the basal turn length using the $\mathrm{A}$ value to reach a final linear equation, providing a reliable method to calculate the CDL using a single CT scan parameter in a time-efficient manner. The CDL calculated with the most accurate method, that is, 3D reconstruction, comes to a mean of $35.04 \mathrm{~mm}$ at the level of the organ of Corti, ${ }^{10-13}$ and of $37.07 \mathrm{~mm}$ measured at the lateral wall of the cochlea. $7,11,13,15$

There are few studies reporting on CDL data in the Asian population. Thong et $\mathrm{al}^{8}$ calculated the length of the basal turn of the cochlea in a population sample comprising of Chinese, Malay and Indian patients using Escudé's formula in HRCT of the temporal bones, and reported that significant ethnic and gender variations exist in the length of the cochlear basal turn.
They reported the length of the basal turn of the cochlea to be of $22.51 \mathrm{~mm}$ and $21.81 \mathrm{~mm}$ in Chinese males and females, of $22.39 \mathrm{~mm}$ and $21.88 \mathrm{~mm}$ in Malay males and females, and of $21.93 \mathrm{~mm}$ and $21.81 \mathrm{~mm}$ in Indian males and females respectively. Grover et $\mathrm{al}^{9}$ reported the average length of the cochlea in Indian patients to be of $29.8 \mathrm{~mm}$ (range: $28 \mathrm{~mm}$ to $34.3 \mathrm{~mm}$ ) in a pediatric population younger than 6 years old being evaluated for cochlear implantation. Our data in cadaveric adult temporal bones yielded a mean value of $30.7 \mathrm{~mm}$ (range: $27.6 \mathrm{~mm}$ to $33.4 \mathrm{~mm}$ ) without any significant difference between the two sides. As shown by Yukawa et $\mathrm{al}^{16}$ and Finley et $\mathrm{al}^{17}$ the differential lengths of the electrodes inserted inside cochlear lumen result in stimulation of the tonotopically-arranged frequency-sensing apparatus to variable degrees to the spectral information as a result of frequency map mismatch, resulting in significantly different word recognition capability on the part of the implantees.

Our study has some limitations, including the inability to analyze gender-based variability in CDL and the exact age parameters of the cadavers. The inclusion of only the adult population in the analysis of the CDL is another factor. However, it is well described in the literature that the length/height of the adult cochlea does not change after birth; ${ }^{18,19}$ hence, the age factor should not preclude extending the results to the pediatric population. The results of our study are in unison with the previously-published studies in the literature ${ }^{8,9}$ documenting the ethnic variability in the CDL. It would be relevant and convenient to incorporate the calculated $\mathrm{CDL}$ derived from preoperative HRCT scans of the temporal bone in the standard reporting document, to guide the surgeon inserting the electrode in a customized fashion to ensure better postoperative outcomes in terms of acoustic and speech abilities and preservation of residual hearing. 


\section{Conclusion}

The CDL calculated in the present cadaveric temporal bone study was of $30.7 \mathrm{~mm}$ on average, with no significant side difference. The preoperative HRCT of the temporal bone can be used to calculate the length of the basal turn of cochlea and the CDL to gauge the electrode insertion depth in a customized manner in a particular patient. This can serve as a leading piece of information intraoperatively to maintain the congruity of the frequency-matching between the spectral information input and the processing by the cochlear machinery to result in effective acoustic/speech perception, given the high degree of variability among various populations.

\section{Conflict of Interests}

The authors have no conflict of interests to declare.

\section{References}

1 Hardy M. The length of the organ of Corti in man. Am J Anat 1938; 62:291-311

2 Bredberg G. Cellular pattern and nerve supply of the human organ of Corti. Acta Otolaryngol 1968(Suppl 236):1+

3 Retzius G. Das gehororgan der werbelthiere. Morphologisch histologische studien. Samsen and Waalin, Stockholm. Vol I 1881;II:1884

4 Alexiades G, Dhanasingh A, Jolly C. Method to estimate the complete and two-turn cochlear duct length. Otol Neurotol 2015;36(05): 904-907

5 Blake S. Wilson, Michael F. Dorman, The design of cochlear implants. In: John K. Niparko, Editor. Cochlear Implants: Principles \& Practices. $2^{\text {nd }}$ edition. Philadelphia: Lippincott Williams \& Wilkins; 2009:97

6 Ulehlová L, Voldřich L, Janisch R. Correlative study of sensory cell density and cochlear length in humans. Hear Res 1987;28(23):149-151

7 Würfel W, Lanfermann H, Lenarz T, Majdani O. Cochlear length determination using Cone Beam Computed Tomography in a clinical setting. Hear Res 2014;316:65-72
8 Thong JF, Low D, Tham A, Liew C, Tan TY, Yuen HW. Cochlear duct length-one size fits all? Am J Otolaryngol 2017;38(02): 218-221

9 Grover M, Sharma S, Singh SN, Kataria T, Lakhawat RS, Sharma MP. Measuring cochlear duct length in Asian population: worth giving a thought!. Eur Arch Otorhinolaryngol 2018;275(03):725-728

10 Sato H, Sando I, Takahashi H. Sexual dimorphism and development of the human cochlea. Computer 3-D measurement. Acta Otolaryngol 1991;111(06):1037-1040

11 Koch RW, Ladak HM, Elfarnawany M, Agrawal SK. Measuring Cochlear Duct Length - a historical analysis of methods and results. J Otolaryngol Head Neck Surg 2017;46(01):19

12 Takagi A, Sando I. Computer-aided three-dimensional reconstruction: a method of measuring temporal bone structures including the length of the cochlea. Ann Otol Rhinol Laryngol 1989;98(7 Pt 1):515-522

13 Kawano A, Seldon HL, Clark GM. Computer-aided three-dimensional reconstruction in human cochlear maps: measurement of the lengths of organ of Corti, outer wall, inner wall, and Rosenthal's canal. Ann Otol Rhinol Laryngol 1996;105(09): 701-709

14 Escudé B, James C, Deguine O, Cochard N, Eter E, Fraysse B. The size of the cochlea and predictions of insertion depth angles for cochlear implant electrodes. Audiol Neurotol 2006;11(Suppl 1):27-33

15 Meng J, Li S, Zhang F, Li Q Qin Z. Cochlear size and shape variability and implications in cochlear implantation surgery. Otol Neurotol 2016;37(09):1307-1313

16 Yukawa K, Cohen L, Blamey P, Pyman B, Tungvachirakul V, O'Leary S. Effects of insertion depth of cochlear implant electrodes upon speech perception. Audiol Neurotol 2004;9(03): 163-172

17 Finley CC, Holden TA, Holden LK, et al. Role of electrode placement as a contributor to variability in cochlear implant outcomes. Otol Neurotol 2008;29(07):920-928

18 Mori MC, Chang KW. CT analysis demonstrates that cochlear height does not change with age. AJNR Am J Neuroradiol 2012; 33(01):119-123

19 Pelliccia P, Venail F, Bonafé A, et al. Cochlea size variability and implications in clinical practice. Acta Otorhinolaryngol Ital 2014; 34(01):42-49 